\title{
SOBOLEV MAPPING OF SOME HOLOMORPHIC PROJECTIONS
}

\author{
L. D. EDHOLM \& J. D. MCNEAL
}

\begin{abstract}
Sobolev irregularity of the Bergman projection on a family of domains containing the Hartogs triangle is shown. On the Hartogs triangle itself, a sub-Bergman projection is shown to satisfy better Sobolev norm estimates than its Bergman projection.
\end{abstract}

\section{Introduction}

If $\Omega \subset \mathbb{C}^{n}$ is an open set, $1<p<\infty$, and $k \in \mathbb{Z}^{+}$, let $L_{k}^{p}(\Omega)$ denote the usual $L^{p}$ Sobolev space of order $k$ : the measurable functions $f$ such that

$$
\|f\|_{L_{k}^{p}(\Omega)}=\left(\sum_{|\alpha| \leq k} \int_{\Omega}\left|\partial^{\alpha} f\right|^{p} d V\right)^{\frac{1}{p}}
$$

is finite, where derivatives are interpreted in the distributional sense.

This paper continues investigations from [19], 20] by demonstrating irregularity in the $L^{p}$ Sobolev spaces for the Bergman projection associated to domains defined in (1.2) below. These generalize the Hartogs triangle, which is $\mathbb{H}_{1}$ in (1.2).

The Bergman projection, $\boldsymbol{B}=\boldsymbol{B}_{\Omega}$, orthogonally projects $L^{2}(\Omega)$ onto the closed subspace $\mathcal{O}(\Omega) \cap L^{2}(\Omega), \mathcal{O}(\Omega)$ denoting holomorphic functions. On $L^{2}(\Omega)=L_{0}^{2}(\Omega), \boldsymbol{B}$ is represented as an integral operator

$$
\boldsymbol{B} f(z)=\int_{\Omega} B_{\Omega}(z, w) f(w) d V(w), \quad f \in L^{2}(\Omega),
$$

where $d V$ denotes Lebesgue measure and $B_{\Omega}(z, w) \in \mathcal{O}(\Omega) \times \overline{\mathcal{O}(\Omega)}$ is the Bergman kernel. If $f \notin L^{2}(\Omega)$ let (1.1) define $\boldsymbol{B} f$, whenever the integral converges. For many classes of pseudoconvex domains, precise pointwise estimates on $B_{\Omega}(z, w)$ were obtained and shown to imply $\|\boldsymbol{B} f\|_{L_{k}^{p}(\Omega)} \leq C\|f\|_{L_{k}^{p}(\Omega)}$ for all $1<p<\infty$ and $k \in \mathbb{Z}^{+}$. See [12, 23, 25, 26, 27, 30, 31]. Thus $\boldsymbol{B}$ is $L_{k}^{p}$-regular in these cases. In the special case $p=2$, regularity for all $k \in \mathbb{Z}^{+}$was shown in [8] whenever $\Omega$ has a plurisubharmonic defining function, without establishing pointwise estimates on $B_{\Omega}(z, w)$. This result was generalized in [9, 24]. However $L_{k}^{2}$ regularity does not always hold. This irregularity was discovered in connection to Condition $R$ of Bell-Ligocka [5, 6]: it is shown in [4] that $\boldsymbol{B}$ is irregular on $L_{k}^{2}(W)$, for large $k$, on the pseudoconvex "worm" domains $W$ given in [16].

The irregularity of $\boldsymbol{B}$ demonstrated in [19, 20] is somewhat different. It occurs on the Lebesgue spaces $L^{p}(\Omega)=L_{0}^{p}(\Omega)$ for certain $p \neq 2$ and does not involve derivatives. For $\gamma>0$, define

$$
\mathbb{H}_{\gamma}=\left\{\left(z_{1}, z_{2}\right) \in \mathbb{C}^{2}:\left|z_{1}\right|^{\gamma}<\left|z_{2}\right|<1\right\} .
$$

In [20] it is shown that the Bergman projection on $\mathbb{H}_{\gamma}$, for any $\gamma$, is a degenerate $L^{p}$ operator, bounded only for $p$ in a proper subinterval of $(1, \infty)$. In particular the situation on $\mathbb{H}_{1}$ is that $\boldsymbol{B}: L^{p}\left(\mathbb{H}_{1}\right) \rightarrow L^{p}\left(\mathbb{H}_{1}\right)$ boundedly if and only if $\frac{4}{3}<p<4$; see Theorem 3.1 below for the general situation. The limited range of $L^{p}$ boundedness has consequences

2010 Mathematics Subject Classification. 32A36, 32A25, 32W05. 
for approximation and duality theory in $\mathcal{O}\left(\mathbb{H}_{\gamma}\right)$, see [10. Similar consequences hold when irregularity can be characterized on norm scales other than $L^{p}$.

It turns out $\boldsymbol{B}$ is very degenerate as an $L^{p}$ Sobolev map.

Theorem 1.3. Let $\gamma=\frac{m}{n} \in \mathbb{Q}^{+}$and $\boldsymbol{B}$ denote the Bergman projection on $\mathbb{H}_{\gamma}$.

(1) $\boldsymbol{B}$ fails to map $L_{k}^{2}\left(\mathbb{H}_{\gamma}\right) \rightarrow L_{k}^{2}\left(\mathbb{H}_{\gamma}\right)$, for $k \geq 1$ an integer.

(2) Let $j, l \in \mathbb{Z}^{+}$. Then $\frac{\partial^{j+l}}{\partial z_{1}^{j} \partial z_{2}^{l}} \circ \boldsymbol{B}$ fails to map $C^{\infty}\left(\overline{\mathbb{H}_{\gamma}}\right) \rightarrow L^{p}\left(\mathbb{H}_{\gamma}\right)$ for

$$
p \geq \frac{2 m+2 n}{m(l+1)+n(j+1)-1} .
$$

$\boldsymbol{B}$ also exhibits some regularity in $L^{p}$ Sobolev norms, but only on $\mathbb{H}_{1}$ :

Theorem 1.4. $\boldsymbol{B}$ maps $L_{1}^{p}\left(\mathbb{H}_{1}\right) \rightarrow L_{1}^{p}\left(\mathbb{H}_{1}\right)$ boundedly, for $\frac{4}{3}<p<2$.

Notice the range on $p$, for boundedness on $L_{1}^{p}\left(\mathbb{H}_{1}\right)$, is smaller than the range for boundedness on $L_{0}^{p}\left(\mathbb{H}_{1}\right)$. More general statements than Theorem 1.4 can be made - for separate directional derivatives and on domains other than $\mathbb{H}_{1}$ - but these do not yield boundedness theorems on the full Sobolev spaces $L_{k}^{p}$; see Section 4 .

Proving Theorems 1.3 and 1.4 requires understanding how derivatives commute past the Bergman projection. An initial difficulty is that $\mathbb{H}_{\gamma}$ is not smoothly bounded, so Stokes' theorem cannot be applied in the usual way, e.g., as in [28, Lemma 3], [29, Proposition 3.3], or [30, Lemma 5.1]. We circumvent this by applying Stokes theorem on appropriately chosen discs and annuli intersecting $\mathbb{H}_{\gamma}$. Theorem 1.3 is proved in Section 3, After developing some general tools, Theorem 1.4 is proved as Corollary 4.19 in Section 4, To partially repair irregularity described by Theorem 1.3, substitute operators related to $\boldsymbol{B}$ are considered in Section 5 .

There are other papers showing Bergman irregularity on $L_{0}^{p}(\Omega)$, for specific pseudoconvex $\Omega:$ [1, 11, 13, 34]. A unifying result, explaining irregularity in these cases and [19, 20], is lacking. A weighted regularity result on $L_{k}^{p}\left(\mathbb{H}_{1}\right), k>0$, related to Theorem 1.3, was obtained in [14]. See also the paper [2] for a nonpseudoconvex domain with $L_{0}^{p}$-irregularity of its Bergman projection.

When $X$ and $Y$ are expressions involving several variables, write $X \lesssim Y$ to mean $X \leq C Y$ for a constant $C$ independent of certain of these variables. The independence of which variables is specified in use. $X \approx Y$ means $X \lesssim Y \lesssim X$ holds.

\section{Sobolev Regularity in ONE VARIAble}

Let $D \subset \mathbb{C}$ denote the unit disc. The Bergman projection $\boldsymbol{B}_{D}$ is bounded from $L_{k}^{p}(D) \rightarrow$ $L_{k}^{p}(D)$ for all $1<p<\infty$ and $k \in \mathbb{Z}^{+}$. This is well-known when $k=0$, apparently first proved in [33] using singular integral operator theory; see [17, Chapter 2]. For any $k \in \mathbb{Z}^{+}$, a proof modeled on arguments in [28] is given below. This serves as a template for the proof of Theorem 1.4.

The Bergman kernel of $D$ is

$$
B_{D}(z, w)=\frac{1}{\pi} \sum_{j=0}^{\infty}(j+1)(z \bar{w})^{j}=\frac{1}{\pi} \frac{1}{(1-z \bar{w})^{2}} .
$$

Note $B_{D}(z, w)$ can be viewed as a function of $s=z \bar{w}$. 
2.1. $L_{0}^{p}$ boundedness. A family of integral estimates will be used. When $A=0$, the result is often called the Forelli-Rudin lemma; see [21, [32, or [35] for the 'standard' proof, based on asymptotics of the gamma function. Different proofs are given in [19], [20, [13], which also address $A \neq 0$.

Lemma 2.2. Let $D \subset \mathbb{C}$ be the unit disc, $\epsilon \in(0,1)$ and $A<2$. Then for $z \in D$,

$$
\int_{D} \frac{\left(1-|w|^{2}\right)^{-\epsilon}}{|1-z \bar{w}|^{2}}|w|^{-A} d V(w) \lesssim\left(1-|z|^{2}\right)^{-\epsilon}
$$

for a constant $C=C(A, \epsilon)$ independent of $z$.

A general version of Schur's Lemma will also be used. The next result extends Lemma 2.4 from [19].

Lemma 2.3. Let $\Omega \subset \mathbb{C}^{n}$ be a domain and $K: \Omega \times \Omega \rightarrow[0, \infty)$ a kernel function. Suppose there is an auxiliary function $h: \Omega \rightarrow[0, \infty)$ and numbers $0 \leq \alpha<\beta, 0 \leq \gamma<\delta$ such that the following two estimates hold: For all $\epsilon \in[\alpha, \beta)$,

$$
\int_{\Omega} K(z, w) h(w)^{-\epsilon} d V(w) \lesssim h(z)^{-\epsilon}
$$

and for all $\epsilon \in[\gamma, \delta)$,

$$
\int_{\Omega} K(z, w) h(z)^{-\epsilon} d V(z) \lesssim h(w)^{-\epsilon}
$$

Then the operator $\mathcal{K}, \mathcal{K}(f)(z):=\int_{\Omega} K(z, w) f(w) d V(w)$, maps $L^{p}(\Omega) \rightarrow L^{p}(\Omega)$ for all $p$ in the range

$$
\frac{\gamma}{\beta}+1<p<\frac{\delta}{\alpha}+1
$$

Proof. Let $\frac{1}{p}+\frac{1}{q}=1, g \in L^{p}(\Omega)$ and $s \in[\alpha, \beta)$. Then

$$
\begin{aligned}
|\mathcal{K}(f)(z)|^{p} & \leq\left(\int_{\Omega} K(z, w)|f(w)|^{p} h(w)^{\frac{s p}{q}} d V(w)\right)\left(\int_{\Omega} K(z, w) h(w)^{-s} d V(w)\right)^{\frac{p}{q}} \\
& \lesssim\left(\int_{\Omega} K(z, w)|f(w)|^{p} h(w)^{\frac{s p}{q}} d V(w)\right) h(z)^{-\frac{s p}{q}} .
\end{aligned}
$$

The first inequality follows from Hölder's inequality, the second from (2.4). Now

$$
\begin{aligned}
\int_{\Omega}|\mathcal{K}(f)(z)|^{p} d V(z) & \lesssim \int_{\Omega}\left(\int_{\Omega} K(z, w)|f(w)|^{p} h(w)^{\frac{s p}{q}} d V(w)\right) h(z)^{-\frac{s p}{q}} d V(z) \\
& =\int_{\Omega}|f(w)|^{p} h(w)^{\frac{s p}{q}}\left(\int_{\Omega} K(z, w) h(z)^{-\frac{s p}{q}} d V(z)\right) d V(w) .
\end{aligned}
$$

When $s \in[\alpha, \beta)$ may chosen so that also $\frac{s p}{q} \in[\gamma, \delta)$, estimate (2.5) implies

$$
(2.7) \lesssim \int_{\Omega}|f(z)|^{p} d V(z)
$$

and thus $\mathcal{K}: L^{p}(\Omega) \rightarrow L^{p}(\Omega)$ boundedly. The existence of such an $s$ is equivalent to saying both the inequalities $\frac{q}{p} \gamma<\beta$ and $\alpha<\frac{q}{p} \delta$ hold. This is equivalent to saying (2.6) holds, as claimed.

Lemmas 2.2 and 2.3 suffice to show $L_{0}^{p}(D)$ boundedness of $\boldsymbol{B}_{D}$.

Corollary 2.8. The Bergman projection $\boldsymbol{B}_{D}$ maps $L^{p}(D)$ to $L^{p}(D)$ for all $1<p<\infty$.

In fact, the operator whose kernel is $\left|B_{D}(z, w)\right|$ is bounded on $L^{p}(D)$ for $1<p<\infty$. 
Proof. Lemma 2.3 is used with $K(z, w)=\left|B_{D}(z, w)\right|$ and $h(w)=1-|w|^{2}$ as the auxiliary function. Lemma 2.2 shows that estimate (2.4) holds for all $0<\epsilon<1$. Since $B_{D}(z, w)$ is conjugate symmetric, (2.4) is equivalent to (2.5) with $\alpha=\gamma$ and $\beta=\delta$. Lemma 2.3 then gives the claimed boundedness by setting $\beta=1$ and sending $\alpha \rightarrow 0^{+}$.

2.2. Integration by parts. Define the vector field

$$
\mathcal{T}_{w}=\bar{w} \frac{\partial}{\partial \bar{w}}-w \frac{\partial}{\partial w}
$$

and write $\mathcal{T}_{w}^{k}$ to mean $\mathcal{T}_{w} \circ \cdots \circ \mathcal{T}_{w}$ composed $k$ times. If $f \in L_{k}^{p}(D)$, clearly $\mathcal{T}_{w}^{k} f \in L^{p}(D)$. If, in addition, $f \in \mathcal{O}(D)$, a partial converse holds: if $D_{\delta}=\{z \in D:|z|>\delta\}$, then $\|f\|_{L_{k}^{p}\left(D_{\delta}\right)} \lesssim\left\|\mathcal{T}_{w}^{k} f\right\|_{L^{p}\left(D_{\delta}\right)}$ for a constant independent of $f \mathbb{1}$ This holds since any first derivative can be written as a linear combination $A \mathcal{T}_{w}+B \frac{\partial}{\partial \bar{w}}$ on $D_{\delta}$, for bounded functions $A$ and $B$.

The crucial property $\mathcal{T}_{w}$ satisfies is

Proposition 2.10. $\mathcal{T}_{w}$ annihilates $C^{1}$ radial functions of $w \in \mathbb{C}$.

Proof. A $C^{1}$ radial function $g$ can be written as $g(w)=f\left(|w|^{2}\right)$, where $f \in C^{1}([0, \infty))$. Therefore

$$
\mathcal{T}_{w} g=\bar{w} f^{\prime}\left(|w|^{2}\right) \cdot w-w f^{\prime}\left(|w|^{2}\right) \cdot \bar{w} \equiv 0
$$

Recall that $r: \mathbb{C} \rightarrow \mathbb{R}$ is a defining function for $\Omega$ if $\{r<0\}=\Omega$ and $|\nabla r(w)| \neq 0$ when $r=0$. Proposition 2.10 implies, in particular, that $\mathcal{T}_{w}$ annihilates defining functions of discs and annuli centered at the origin along their boundaries. An integration by parts result follows:

Proposition 2.11. Let $\Omega \subset \mathbb{C}$ be either a disc or an annulus centered at the origin. Then if $f, g \in L_{1}^{1}(\Omega) \cap C(\bar{\Omega})$,

$$
\int_{\Omega} \mathcal{T}_{w} f \cdot g d V=-\int_{\Omega} f \cdot \mathcal{T}_{w} g d V
$$

Proof. Choose a defining function for $\Omega$ with $|\nabla r(w)|=1$ for all $w \in b \Omega$. Stokes' theorem yields

$$
\begin{aligned}
\int_{\Omega} \mathcal{T}_{w} f \cdot g d V= & \int_{\Omega} \frac{\partial f}{\partial \bar{w}}(w) \cdot \bar{w} g(w) d V(w)-\int_{\Omega} \frac{\partial f}{\partial w}(w) \cdot w g(w) d V(w) \\
= & -\int_{\Omega} f \cdot \frac{\partial}{\partial \bar{w}}(\bar{w} g(w)) d V+\int_{b \Omega} f \cdot \bar{w} g \cdot \frac{\partial r}{\partial \bar{w}} d S \\
& \quad \quad \int_{\Omega} f \cdot \frac{\partial}{\partial w}(w g(w)) d V-\int_{b \Omega} f w g \cdot \frac{\partial r}{\partial w} d S \\
= & -\int_{\Omega} f \cdot\left[\frac{\partial}{\partial \bar{w}}(\bar{w} g(w))-\frac{\partial}{\partial w}(w g(w))\right] d V+\int_{b \Omega} f g \cdot \mathcal{T}_{w} r(w) d S \\
= & -\int_{\Omega} f \cdot \mathcal{T}_{w} g d V .
\end{aligned}
$$

Here $d S$ denotes induced surface measure on $b \Omega$. The last boundary integral vanishes since $\mathcal{T}_{w} r \equiv 0$ on $b \Omega$.

\footnotetext{
${ }^{1} \mathrm{~A}$ version of this also holds in several variables. See, e.g., 7, 3, 22, for a statement of the result, as well as elementary proofs for $p=2$. For general $p$, see [15].
} 


\section{3. $L_{k}^{p}$ boundedness for $k>0$.}

Theorem 2.12. The Bergman projection $\boldsymbol{B}_{D}$ is a bounded operator from $L_{k}^{p}(D) \rightarrow L_{k}^{p}(D)$ for all $k \in \mathbb{Z}^{+}$and $1<p<\infty$.

Proof. Fix $k, p$ and let $f \in L_{k}^{p}(D)$. Since $\boldsymbol{B}_{D} f \in \mathcal{O}(\Omega)$, only holomorphic derivatives need to be estimated. For $z \neq 0$,

$$
\begin{aligned}
\frac{\partial^{k}}{\partial z^{k}} \boldsymbol{B}_{D} f(z) & =\frac{\partial^{k}}{\partial z^{k}} \int_{D} B_{D}(z, w) f(w) d V(w) \\
& =\int_{D} \frac{\partial^{k}}{\partial z^{k}}\left(B_{D}(z, w)\right) f(w) d V(w) \\
& =\frac{1}{z^{k}} \int_{D} \bar{w}^{k} \frac{\partial^{k}}{\partial \bar{w}^{k}}\left(B_{D}(z, w)\right) f(w) d V(w) .
\end{aligned}
$$

The last equality follows because $B_{D}(z, w)$ can be viewed as a function of the variable $s=z \bar{w}$. Define a new kernel $K_{k}(z, w)$, obtained by subtracting away the $(k-1)$-Taylor approximation of $B_{D}(z, w)$ in the $s$ variable, i.e.,

$$
\begin{aligned}
K_{k}(z, w):=\frac{1}{\pi^{2}}\left[\frac{1}{(1-s)^{2}}-\sum_{j=0}^{k-1}(j+1) s^{j}\right] & =\frac{1}{\pi^{2}} \frac{\partial}{\partial s}\left[\sum_{j=k}^{\infty} s^{j+1}\right] \\
& =\frac{(k+1) s^{k}-k s^{k+1}}{(1-s)^{2}} .
\end{aligned}
$$

Since $K_{k}(z, w)$ and $B_{D}(z, w)$ differ by terms annihilated by $\frac{\partial^{k}}{\partial \bar{w}^{k}}$ and $K_{k}(z, w)$ is antiholomorphic in $w$,

$$
\begin{aligned}
(2.13) & =\frac{1}{z^{k}} \int_{D} \bar{w}^{k} \frac{\partial^{k}}{\partial \bar{w}^{k}}\left(K_{k}(z, w)\right) f(w) d V(w) \\
& =\frac{1}{z^{k}} \int_{D} \mathcal{T}_{w}^{k}\left(K_{k}(z, w)\right) f(w) d V(w), \\
& =\frac{(-1)^{k}}{z^{k}} \int_{D} K_{k}(z, w) \mathcal{T}_{w}^{k} f(w) d V(w) .
\end{aligned}
$$

The last equality follows from Proposition 2.11,

The modified kernel $K_{k}(z, w)$ satisfies a stronger estimate than $B_{D}(z, w)$. Indeed, equation (2.14) shows

$$
\left|K_{k}(z, w)\right| \lesssim \frac{|z|^{k}|w|^{k}}{|1-z \bar{w}|^{2}}
$$

for a constant independent of $z, w \in D$. This can be used to counteract the factor $\frac{1}{z^{k}}$ appearing in (2.13). Thus

$$
\begin{aligned}
\left|\frac{\partial^{k}}{\partial z^{k}} \boldsymbol{B}_{D} f(z)\right| \lesssim \int_{D} \frac{|w|^{k}}{|1-z \bar{w}|^{2}} \mathcal{T}_{w}^{k} f(w) d V(w) & \leq \int_{D} \frac{1}{|1-z \bar{w}|^{2}} \mathcal{T}_{w}^{k} f(w) d V(w) \\
& \approx \int_{D}\left|B_{D}(z, w)\right| \mathcal{T}_{w}^{k} f(w) d V(w) .
\end{aligned}
$$

Since $\mathcal{T}_{w}^{k} f \in L^{p}(D)$, Corollary 2.8 says that (2.15) defines an $L^{p}(D)$ function. This implies $\frac{\partial^{k}}{\partial z^{k}} \boldsymbol{B}_{D} f(z) \in L^{p}(D)$.

For any positive integer $l \leq k$, the same argument - but for the modified kernel $K_{l}(z, w)$ - shows $\frac{\partial^{l}}{\partial z^{l}} \boldsymbol{B}_{D} f(z) \in L^{p}(D)$. Thus $\boldsymbol{B}_{D} f \in L_{k}^{p}(D)$. 


\section{SOBOlEV IRREgularity}

The starting point is the characterization of $L_{0}^{p}$ boundedness of the Bergman projection on $\mathbb{H}_{\gamma}$.

Theorem $3.1([20])$. Let $\mathbb{H}_{\gamma}$ be defined in (1.2), B denote the Bergman projection on $\mathbb{H}_{\gamma}$, and $1<p<\infty$.

(1) Let $\gamma=\frac{m}{n} \in \mathbb{Q}^{+}$, with $\operatorname{gcd}(m, n)=1$.

Then $\boldsymbol{B}: L^{p}\left(\mathbb{H}_{\gamma}\right) \rightarrow L^{p}\left(\mathbb{H}_{\gamma}\right)$ is bounded if and only if $p \in\left(\frac{2 m+2 n}{m+n+1}, \frac{2 m+2 n}{m+n-1}\right)$.

(2) Let $\gamma>0$ be irrational.

Then $\boldsymbol{B}: L^{p}\left(\mathbb{H}_{\gamma}\right) \rightarrow L^{p}\left(\mathbb{H}_{\gamma}\right)$ is bounded if and only if $p=2$.

Let $(\lambda(m, n), \rho(m, n))=\left(\frac{2 m+2 n}{m+n+1}, \frac{2 m+2 n}{m+n-1}\right)$ denote the interval of $L^{p}$ boundedness in (1) above. When $\mathbb{H}_{m / n}$ is fixed, denote this also as $I_{0}^{p}$.

Some ingredients in the proof of Theorem 3.1 are used to prove the irregularity statements in Theorem 1.3. Only consider $\mathbb{H}_{m / n}$ and let $\boldsymbol{B}=\boldsymbol{B}_{\mathbb{H}_{m / n}}$. An index $\left(\alpha_{1}, \alpha_{2}\right) \in \mathbb{Z}^{+} \times \mathbb{Z}$ is $L^{p}$-allowable if the monomial $z_{1}^{\alpha_{1}} \tilde{z}_{2}^{\alpha_{2}} \in L^{p}\left(\mathbb{H}_{m / n}\right)$, where $\tilde{z}_{2}$ is either $z_{2}$ or $\bar{z}_{2}$. This set can be characterized:

Lemma $3.2\left([20]\right.$, eq. (3.3)). Let $p \in[1, \infty)$. The $L^{p}$-allowable indices are

$$
\mathcal{S}\left(\mathbb{H}_{m / n}, L^{p}\right)=\left\{\alpha=\left(\alpha_{1}, \alpha_{2}\right): \alpha_{1} \geq 0, n \alpha_{1}+m \alpha_{2} \geq\left\lfloor-\frac{2}{p}(m+n)+1\right\rfloor\right\} .
$$

See also Lemma 4.4 in [10]. Here $\lfloor x\rfloor=$ the greatest integer $\leq x$. In particular, the $L^{2}$ monomials are

$$
\mathcal{S}\left(\mathbb{H}_{m / n}, L^{2}\right)=\left\{\left(\alpha_{1}, \alpha_{2}\right): \alpha_{1} \geq 0, n \alpha_{1}+m \alpha_{2} \geq-m-n+1\right\} .
$$

As notation for the ray bounding the sets $\mathcal{S}\left(\mathbb{H}_{m / n}, L^{p}\right)$, let

$$
\ell\left(\mathbb{H}_{m / n}, L^{p}\right)=\left\{(x, y) \in \mathbb{R}^{2}: x \geq 0, \quad n x+m y=\left\lfloor-\frac{2}{p}(m+n)+1\right\rfloor\right\} .
$$

A consequence of orthogonality is also essential.

Lemma 3.4 ([20], Proposition 5.1). If both $\left(\beta_{1}, \beta_{2}\right),\left(\beta_{1},-\beta_{2}\right) \in \mathcal{S}\left(\mathbb{H}_{m / n}, L^{2}\right)$, then

$$
\boldsymbol{B}\left(z_{1}^{\beta_{1}} \bar{z}_{2}^{\beta_{2}}\right)=C z_{1}^{\beta_{1}} z_{2}^{-\beta_{2}}
$$

for a constant $C>0$.

The unboundedness statements in Theorem 3.1 for $p \notin I_{0}^{p}$ (defined above) are proved as follows. Let $p \geq \rho(m, n)$.

(A): Choose $\left(\beta_{1}, \beta_{2}\right) \in \mathbb{Z}^{+} \times \mathbb{Z}^{+}$with $\left(\beta_{1},-\beta_{2}\right) \in \ell\left(\mathbb{H}_{m / n}, L^{2}\right)$.

(B): Lemma 3.2 implies $z_{1}^{\beta_{1}} z_{2}^{-\beta_{2}} \notin \mathcal{S}\left(\mathbb{H}_{m / n}, L^{\rho(m, n)}\right)$.

(C): Let $f\left(z_{1}, z_{2}\right)=: z_{1}^{\beta_{1}} \bar{z}_{2}^{\beta_{2}}$; Lemma 3.4 says $\boldsymbol{B} f=C z_{1}^{\beta_{1}} z_{2}^{-\beta_{2}}$. Thus $\|f\|_{L^{p}}<\infty$, while $\|\boldsymbol{B} f\|_{L^{p}}=\infty$.

Duality implies the same conclusion if $p \leq \lambda(m, n)$.

The heart of this argument works on Sobolev spaces. But one piece is not transferable: if $j, l \in \mathbb{Z}^{+}$, the operator $\frac{\partial^{j+l}}{\partial z_{1}^{j} \partial z_{2}^{l}} \circ \boldsymbol{B}$ is not self-adjoint in the $L^{2}$ inner product. As a result, knowing that $\boldsymbol{B}$ is unbounded on $L_{k}^{p}$ does not automatically imply that $\boldsymbol{B}$ is unbounded on $L_{k}^{q}$, where $\frac{1}{p}+\frac{1}{q}=1$. Whether this fact actually allows regularity of $\boldsymbol{B}$ on $L_{k}^{q}$ for small $q$, e.g., $q<\lambda(m, n)$, in cases where $\boldsymbol{B}$ is unbounded on $L_{k}^{p}$ is uncertain. 
However for large $p, L_{k}^{p}$ regularity certainly does not hold:

Theorem 3.5. Let $\gamma=\frac{m}{n} \in \mathbb{Q}^{+}$, and $j, l$ non-negative integers. The operator $\frac{\partial^{j+l}}{\partial z_{1}^{j} \partial z_{2}^{l}} \circ \boldsymbol{B}$ fails to map $C^{\infty}\left(\overline{\mathbb{H}_{\gamma}}\right) \rightarrow L^{p}\left(\mathbb{H}_{\gamma}\right)$ for any

$$
p \geq \frac{2 m+2 n}{m(l+1)+n(j+1)-1} .
$$

Proof. Starting from equation (3.3), choose $\beta=\left(\beta_{1}, \beta_{2}\right) \in \mathbb{Z}^{+} \times \mathbb{Z}^{+}$with $\beta_{1} \geq j$ and $\left(\beta_{1},-\beta_{2}\right) \in \ell\left(\mathbb{H}_{m / n}, L^{2}\right)$, i.e., $n \beta_{1}-m \beta_{2}=1-m-n$. Clearly

$$
\frac{\partial^{j+l}}{\partial z_{1}^{j} \partial z_{2}^{l}}\left(z_{1}^{\beta_{1}} z_{2}^{-\beta_{2}}\right) \approx z_{1}^{\beta_{1}-j} z_{2}^{-\beta_{2}-l} .
$$

To see when this is an $L^{p}$ function, compute

$$
\begin{aligned}
\int_{\mathbb{H}_{\gamma}}\left|z_{1}^{\beta_{1}-j} z_{2}^{-\beta_{2}-l}\right|^{p} d V & =4 \pi^{2} \int_{h_{\gamma}} r_{1}^{p \beta_{1}-p j+1} r_{2}^{-p \beta_{2}-p l+1} d r_{1} d r_{2} \\
& =4 \pi^{2} \int_{0}^{1} r_{2}^{-p \beta_{2}-p l+1} \int_{0}^{r_{2}^{n / m}} r_{1}^{p \beta_{1}-p j+1} d r_{1} d r_{2} \\
& \approx \int_{0}^{1} r_{2}^{-p \beta_{2}-p l+1+p n \beta_{1} / m-p n j / m+2 n / m} d r_{2},
\end{aligned}
$$

where $h_{\gamma}$ is the Reinhardt shadow of $\mathbb{H}_{\gamma}$, i.e., $h_{\gamma}=\mathbb{H}_{\gamma} \cap\left(\mathbb{R}^{\geq 0} \times \mathbb{R}^{\geq 0}\right)$. The integral in (3.8) is finite if and only if the exponent on the integrand $>-1$. This is equivalent to saying

$$
p<\frac{2 m+2 n}{m(l+1)+n(j+1)-1} .
$$

Now consider the monomial $f(z)=z_{1}^{\beta_{1}} \bar{z}_{2}^{\beta_{2}} \in C^{\infty}\left(\overline{\mathbb{H}_{\gamma}}\right)$. Lemma 3.4 says $\boldsymbol{B} f=C z_{1}^{\beta_{1}} z_{2}^{-\beta_{2}}$. Thus $\|f\|_{L^{p}}<\infty$, while $\left\|\frac{\partial^{j+l}}{\partial z_{1}^{j} \partial z_{2}^{l}} \circ \boldsymbol{B} f\right\|_{L^{p}}=\infty$ for those $p$ satisfying (3.6) .

Remark 3.10. Theorem 3.5 recovers the $L_{0}^{p}$ unboundedness range given in Theorem 3.1 part (1). When $j=l=0$, the right hand side of (3.6) is simply $\rho(m, n)$. Since $\boldsymbol{B}$ is self-adjoint, it must also be unbounded for $1<p<\lambda(m, n)$.

In particular, Theorem 3.5 implies Theorem 1.3 from the Introduction.

Corollary 3.11. Let $k \geq 1$ be an integer and $\gamma=\frac{m}{n} \in \mathbb{Q}^{+}$. The Bergman projection $\boldsymbol{B}$ fails to $\operatorname{map} L_{k}^{2}\left(\mathbb{H}_{\gamma}\right) \rightarrow L_{k}^{2}\left(\mathbb{H}_{\gamma}\right)$.

Proof. If $k=j+l \geq 1$, then $m(l+1)+n(j+1)-1>m+n$. 
The notion of a lattice point diagram associated to the domains $\mathbb{H}_{\gamma}$ was introduced in [20]. The diagrams record exponents of all monomials $z_{1}^{\alpha_{1}} z_{2}^{\alpha_{2}} \in \mathcal{S}\left(\mathbb{H}_{m / n}, L^{p}\right)$, as $p$ varies. These diagrams are thus Newton diagrams, but of the entire space $A^{p}\left(\mathbb{H}_{\gamma}\right)=\mathcal{O}\left(\mathbb{H}_{\gamma}\right) \cap L^{p}\left(\mathbb{H}_{\gamma}\right)$ rather than of an individual $f \in A^{p}\left(\mathbb{H}_{\gamma}\right)$. Several lattice point diagrams succinctly illustrate Theorem 3.5 and Corollary 3.11.

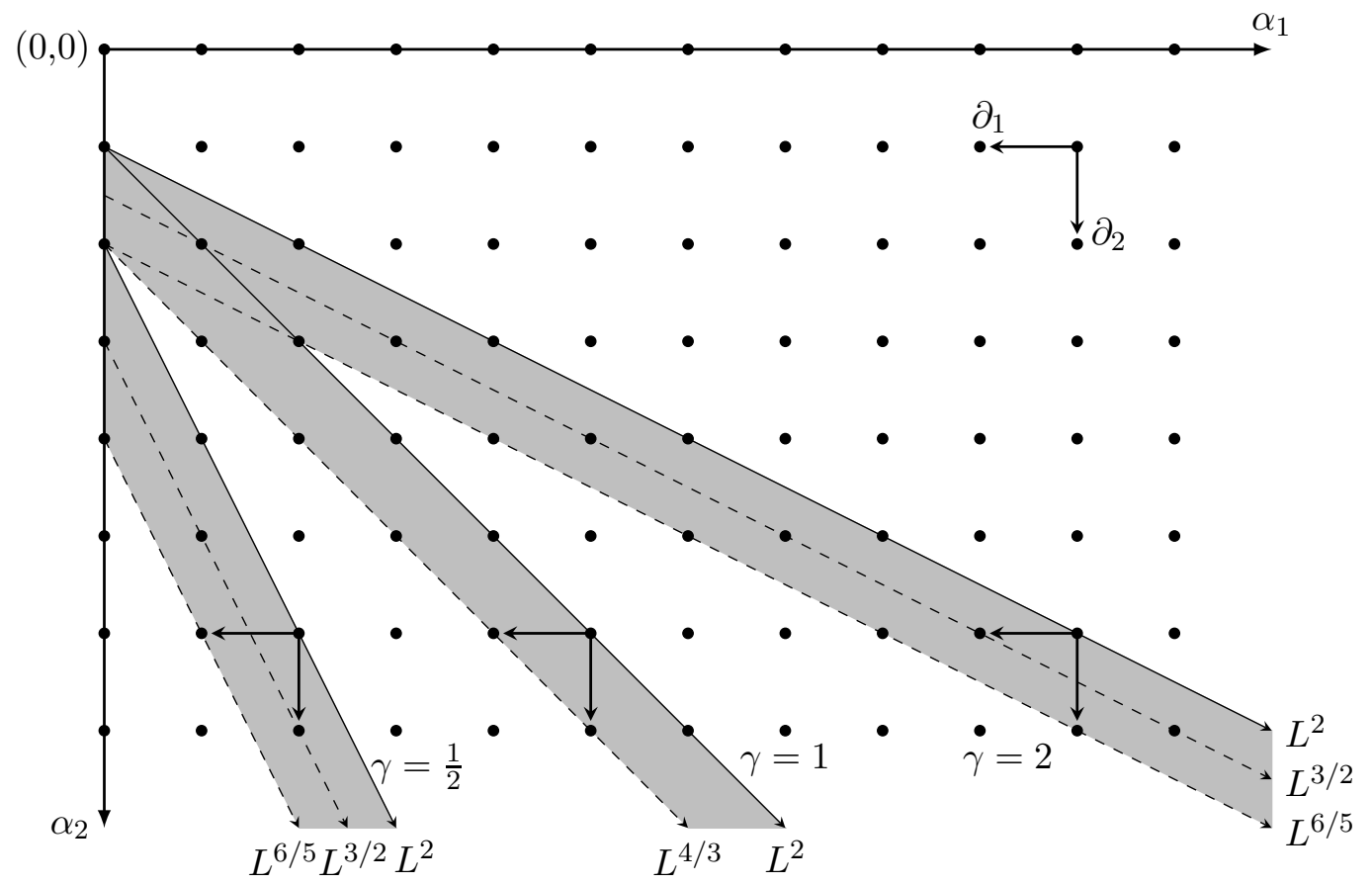

Three lattice point diagrams on $\mathbb{H}_{\gamma}$, corresponding to $\gamma=\frac{m}{n}=\frac{1}{2}, 1,2$, are shown. The indices $\alpha \in \mathcal{S}\left(\mathbb{H}_{m / n}, L^{2}\right)$ are exactly those lattice points on and above the line labeled $L^{2}$ for the corresponding $\gamma$. The dotted lines, labeled $L^{p}$, are lines parallel to their corresponding $L^{2}$ lines but passing through the lattice points in $\ell\left(\mathbb{H}_{m / n}, L^{p}\right)$. Any lattice point strictly below the dotted lines correspond to monomials $\notin L^{p}$ for the given $\mathbb{H}_{m / n}$.

Notice that (up to a constant) $z_{1}$ derivatives of fourth quadrant monomials are represented by a shift left and $z_{2}$ derivatives by a shift down in the lattice point diagram. These operations are labeled $\partial_{1}, \partial_{2}$ in the diagram. The content of Corollary 3.11 is easily seen in this lattice point diagram: monomials on the $L^{2}$ line are driven below to a corresponding $L^{p}$ line $(p<2)$ by a single application of $\partial_{1}$ or $\partial_{2}$. The more precise Theorem 3.5 may also be visualized in this way.

Remark 3.12. The precise non-isotropic (in terms of derivatives) irregularity in Theorem 3.5 seems noteworthy. The two derivative operations $\partial_{1}, \partial_{2}$ are not symmetric with respect to how they drive monomials out of the boundedness interval $I_{0}^{p}$, depending on whether $\gamma>1$ or $\gamma<1$. This is very clear in the diagrams: if $\gamma>1$ (a "fat Hartogs triangle" in the terminology of [18]) more $\partial_{1}$ derivatives are allowed, while if $\gamma<1$ (a "thin Hartogs triangle") more $\partial_{2}$ derivatives are allowed.

\section{Sobolev Regularity}

A class of kernels on the domains $\mathbb{H}_{m / n}$, containing the Bergman kernel $B_{m / n}(z, w)$ and its derivatives, can be analyzed via Lemma 2.3. The next result generalizes Proposition 4.2 of [20], which required $c=d$. 
Lemma 4.1. Let $K: \mathbb{H}_{m / n} \times \mathbb{H}_{m / n} \rightarrow \mathbb{C}$ be an integral kernel satisfying

$$
\left|K\left(z_{1}, z_{2}, w_{1}, w_{2}\right)\right| \lesssim \frac{\left|z_{2}\right|^{c}\left|w_{2}\right|^{d}}{\left|1-z_{2} \bar{w}_{2}\right|^{2}\left|z_{2}^{n} \bar{w}_{2}^{n}-z_{1}^{m} \bar{w}_{1}^{m}\right|^{2}},
$$

and let $\mathcal{K}$ be the operator defined by $\mathcal{K}(f)(z):=\int_{\mathbb{H}_{m / n}} K(z, w) f(w) d V(w)$.

Suppose the following conditions on $c$ and $d$ hold:

$$
c>2 n\left(1-\frac{1}{m}\right)-2, \quad d>2 n\left(1-\frac{1}{m}\right)-2, \quad c+d>2 n\left(2-\frac{1}{m}\right)-2 .
$$

Then $\mathcal{K}: L^{p}\left(\mathbb{H}_{m / n}\right) \rightarrow L^{p}\left(\mathbb{H}_{m / n}\right)$ is bounded operator for all $p \in(1, \infty)$ satisfying

$$
\frac{2 m+2 n}{2 m+2 n+d m-2 m n}<p<\frac{2 m+2 n}{2 m n-c m} .
$$

Remark 4.5. If the exponent $c \geq 2 n$, the upper bound in (4.4) can be taken to be $\infty$. This follows since $\left|z_{2}\right|^{c} \leq\left|z_{2}\right|^{2 n}$ for all $z=\left(z_{1}, z_{2}\right) \in \mathbb{H}_{m / n}$. Similarly if $d \geq 2 n$, the lower bound in (4.4) is 1 .

Note that the conditions in (4.3) are necessary to ensure the range of $p$ in (4.4) is a non-degenerate subinterval of $(1, \infty)$.

Proof of Lemma 4.1. Apply Lemma 2.3, with $h(w)=\left|w_{2}\right|^{R}\left(\left|w_{2}\right|^{2 n}-\left|w_{1}\right|^{2 m}\right)\left(1-\left|w_{2}\right|^{2}\right)$ as the auxiliary function. The parameters $R \geq 0$ and $\epsilon \in(0,1)$ are numbers specified later in the proof. It follows that

$$
\begin{aligned}
(\underline{2.4}) & =\int_{\mathbb{H}_{m / n}}|K(z, w)| h(w)^{-\epsilon} d V(w) \\
& \lesssim \int_{\mathbb{H}_{m / n}} \frac{\left|z_{2}\right|^{c}\left|w_{2}\right|^{d-R \epsilon}\left(\left|w_{2}\right|^{2 n}-\left|w_{1}\right|^{2 m}\right)^{-\epsilon}\left(1-\left|w_{2}\right|^{2}\right)^{-\epsilon}}{\left|1-z_{2} \bar{w}_{2}\right|^{2}\left|z_{2}^{n} \bar{w}_{2}^{n}-z_{1}^{m} \bar{w}_{1}^{m}\right|^{2}} d V(w) \\
& =\int_{D^{*}} \frac{\left|z_{2}\right|^{c}\left|w_{2}\right|^{d-R \epsilon}\left(1-\left|w_{2}\right|^{2}\right)^{-\epsilon}}{\left|1-z_{2} \bar{w}_{2}\right|^{2}}\left[\int_{W} \frac{\left(\left|w_{2}\right|^{2 n}-\left|w_{1}\right|^{2 m}\right)^{-\epsilon}}{\left|z_{2}^{n} \bar{w}_{2}^{n}-z_{1}^{m} \bar{w}_{1}^{m}\right|^{2}} d V\left(w_{1}\right)\right] d V\left(w_{2}\right),
\end{aligned}
$$

where $D^{*}$ is the punctured unit disc and the integral in brackets is taken over the region $W=\left\{w_{1}:\left|w_{1}\right|<\left|w_{2}\right|^{n / m}\right\}$. Denote this inner integral by $I$.

$$
\begin{aligned}
I & =\frac{1}{\left|z_{2}\right|^{2 n}\left|w_{2}\right|^{2 n+2 n \epsilon}} \int_{W}\left(1-\left|\frac{w_{1}^{m}}{w_{2}^{n}}\right|^{2}\right)^{-\epsilon}\left|1-\left(\frac{z_{1}^{m}}{z_{2}^{n}}\right) \overline{\left(\frac{w_{1}^{m}}{w_{2}^{n}}\right)}\right|^{-2} d V\left(w_{1}\right) \\
& =\frac{\left|w_{2}\right|^{2 n / m-2 n-2 n \epsilon}}{m\left|z_{2}\right|^{2 n}} \int_{D} \frac{\left(1-|u|^{2}\right)^{-\epsilon}}{\left|1-z_{1}^{m} z_{2}^{-n} \bar{u}\right|^{2}}|u|^{2 / m-2} d V(u),
\end{aligned}
$$

after the $m$-to-1 integral transformation $u=\frac{w_{1}^{m}}{w_{2}^{n}}$. Lemma 2.2 yields the estimate

$$
\text { (4.7) } \begin{aligned}
\lesssim & \lesssim\left|w_{2}\right|^{2 n / m-2 n-2 n \epsilon}\left(1-\left|\frac{z_{1}^{m}}{z_{2}^{n}}\right|^{2}\right)^{-\epsilon} \\
\left|z_{2}\right|^{2 n} & =\left|z_{2}\right|^{2 n \epsilon-2 n}\left|w_{2}\right|^{2 n / m-2 n-2 n \epsilon}\left(\left|z_{2}\right|^{2 n}-\left|z_{1}\right|^{2 m}\right)^{-\epsilon} .
\end{aligned}
$$

Now insert (4.8) into (4.6):

$$
(4.6) \lesssim\left|z_{2}\right|^{c+2 n \epsilon-2 n}\left(\left|z_{2}\right|^{2 n}-\left|z_{1}\right|^{2 m}\right)^{-\epsilon} \int_{D^{*}} \frac{\left(1-\left|w_{2}\right|^{2}\right)^{-\epsilon}}{\left|1-z_{2} \bar{w}_{2}\right|^{2}}\left|w_{2}\right|^{A} d V\left(w_{2}\right),
$$


where the exponent $A=d+\frac{2 n}{m}-2 n-(2 n+R) \epsilon$ is required to be strictly greater than -2 in order for the $D^{*}$ integral to converge. This is equivalent to requiring

$$
\epsilon<\frac{1}{2 n+R}\left(d+\frac{2 n}{m}-2 n+2\right)
$$

At this stage, fix $R$ large enough to ensure the right hand side of (4.9) $<1$. Lemma 2.2 now applies, since $\epsilon \in(0,1)$. Doing this yields,

$$
\begin{aligned}
\int_{\mathbb{H}_{m / n}}|K(z, w)| h(w)^{-\epsilon} d V(w) & \lesssim\left|z_{2}\right|^{c+2 n \epsilon-2 n}\left(\left|z_{2}\right|^{2 n}-\left|z_{1}\right|^{2 m}\right)^{-\epsilon}\left(1-\left|z_{2}\right|^{2}\right)^{-\epsilon} \\
& <\left|z_{2}\right|^{-R \epsilon}\left(\left|z_{2}\right|^{2 n}-\left|z_{1}\right|^{2 m}\right)^{-\epsilon}\left(1-\left|z_{2}\right|^{2}\right)^{-\epsilon} \\
& =h(z)^{-\epsilon},
\end{aligned}
$$

as long as the exponent $c+2 n \epsilon-2 n>-R \epsilon$. This is equivalent to saying

$$
\epsilon>\frac{2 n-c}{2 n+R}
$$

Inequalities (4.9) and (4.10) give the interval $[\alpha, \beta)$ in Lemma 2.3. Indeed, it suffices to take $\alpha=\frac{2 n-c}{2 n+R}$ and $\beta=\frac{1}{2 n+R}\left(d+\frac{2 n}{m}-2 n+2\right)$.

To generate the interval $[\gamma, \delta)$ needed in Lemma 2.3, simply switch the roles of $c$ and $d$ in the argument above. This leads to taking $\gamma=\frac{2 n-d}{2 n+R}$ and $\delta=\frac{1}{2 n+R}\left(c+\frac{2 n}{m}-2 n+2\right)$. Lemma 2.3 now gives the claimed result.

4.1. Mapping of the differentiated projection. Boundedness of the Bergman projection associated to $\mathbb{H}_{1}$ on the Sobolev space $L_{1}^{p}\left(\mathbb{H}_{1}\right)$ can now be given. In [18], the Bergman kernel of $\mathbb{H}_{1 / n}, n \in \mathbb{Z}^{+}$, is computed as

$$
B_{1 / n}(z, w)=\frac{1}{\pi^{2}} \frac{z_{2}^{n} \bar{w}_{2}^{n}}{\left(1-z_{2} \bar{w}_{2}\right)^{2}\left(z_{2}^{n} \bar{w}_{2}^{n}-z_{1} \bar{w}_{1}\right)^{2}} .
$$

Throughout the section, subscripts on the projection $\boldsymbol{B}_{1 / n}$ and the kernel $B_{1 / n}(z, w)$ are dropped.

Theorem 4.12. On $\mathbb{H}_{1 / n}, n \in \mathbb{Z}^{+}$, it holds that

(1) $\frac{\partial}{\partial z_{1}} \circ \boldsymbol{B}$ maps $L_{1}^{p}\left(\mathbb{H}_{1 / n}\right) \rightarrow L^{p}\left(\mathbb{H}_{1 / n}\right)$ for $p \in\left(1, \frac{2 n+2}{2 n}\right)$.

(2) $\frac{\partial}{\partial z_{2}} \circ \boldsymbol{B}$ maps $L_{1}^{p}\left(\mathbb{H}_{1 / n}\right) \rightarrow L^{p}\left(\mathbb{H}_{1 / n}\right)$ for $p \in\left(\frac{2 n+2}{n+3}, 2\right)$.

Proof. The spirit is similar to the proof of Theorem 2.12, Let $f \in L_{1}^{p}\left(\mathbb{H}_{1 / n}\right)$ for $1<p<\infty$, and $j=1,2$.

$$
\begin{aligned}
\frac{\partial}{\partial z_{j}} \boldsymbol{B} f(z) & =\frac{\partial}{\partial z_{j}} \int_{\mathbb{H}_{1 / n}} B(z, w) f(w) d V(w)=\frac{1}{z_{j}} \int_{\mathbb{H}_{1 / n}} \bar{w}_{j} \frac{\partial}{\partial \bar{w}_{j}}(B(z, w)) f(w) d V(w) \\
& =\frac{1}{z_{j}} \int_{\mathbb{H}_{1 / n}} \mathcal{T}_{w_{j}}(B(z, w)) f(w) d V(w)
\end{aligned}
$$

since $B(z, w)$ is anti-holomorphic in $w$.

The $z_{1}$ and $z_{2}$ derivatives are handled slightly differently. Consider the $z_{2}$ derivative first. Equation (4.13) says

$$
\frac{\partial}{\partial z_{2}} \boldsymbol{B} f(z)=\frac{1}{z_{2}} \int_{\left|w_{1}\right|=0}^{\left|w_{1}\right|=1}\left\{\int_{\mathcal{A}} \mathcal{T}_{w_{2}}(B(z, w)) f(w) d V\left(w_{2}\right)\right\} d V\left(w_{1}\right)
$$


where the inner integral is over $\mathcal{A}=\left\{w_{2}:\left|w_{1}\right|^{1 / n}<\left|w_{2}\right|<1\right\}$ for each fixed $w_{1}$. Since $\mathcal{A}$ is an annulus centered at the origin, Proposition 2.11 transfers the vector field $\mathcal{T}_{w_{2}}$ onto $f$ without picking up a boundary integral:

$$
\begin{aligned}
\text { (4.14) } & =-\frac{1}{z_{2}} \int_{\left|w_{1}\right|=0}^{\left|w_{1}\right|=1}\left\{\int_{\mathcal{A}} B(z, w) \mathcal{T}_{w_{2}} f(w) d V\left(w_{2}\right)\right\} d V\left(w_{1}\right) \\
& =-\frac{1}{z_{2}} \int_{\mathbb{H}_{1 / n}} B(z, w) \mathcal{T}_{w_{2}} f(w) d V(w) \\
& =\int_{\mathbb{H}_{1 / n}} \frac{w_{2}}{z_{2}} B(z, w) \frac{\partial f}{\partial w_{2}}(w) d V(w)-\int_{\mathbb{H}_{1 / n}} \frac{\bar{w}_{2}}{z_{2}} B(z, w) \frac{\partial f}{\partial \bar{w}_{2}}(w) d V(w),
\end{aligned}
$$

derivatives interpreted distributionally. Since $f \in L_{1}^{p}\left(\mathbb{H}_{1 / n}\right), \frac{\partial f}{\partial w_{2}}, \frac{\partial f}{\partial \bar{w}_{2}} \in L^{p}\left(\mathbb{H}_{1 / n}\right)$.

By (4.11), the integral kernels in (4.15) satisfy

$$
\left|\frac{w_{2}}{z_{2}} B(z, w)\right|=\left|\frac{\bar{w}_{2}}{z_{2}} B(z, w)\right| \approx \frac{\left|z_{2}\right|^{n-1}\left|w_{2}\right|^{n+1}}{\left|1-z_{2} \bar{w}_{2}\right|^{2}\left|z_{2}^{n} \bar{w}_{2}^{n}-z_{1} \bar{w}_{1}\right|^{2}} .
$$

Therefore Lemma 4.1, with $c=n-1, d=n+1$, and $m=1$, shows

$$
\left\|\frac{\partial}{\partial z_{2}} \circ \boldsymbol{B} f\right\|_{L^{p}\left(\mathbb{H}_{1 / n}\right)} \lesssim\left\|\frac{\partial f}{\partial w_{2}}\right\|_{L^{p}}+\left\|\frac{\partial f}{\partial \bar{w}_{2}}\right\|_{L^{p}} \leq\|f\|_{L_{1}^{p}\left(\mathbb{H}_{1 / n}\right)}
$$

for $p \in\left(\frac{2 n+2}{n+3}, 2\right)$. This establishes part (2) of the theorem.

Consider the $z_{1}$ derivative. Equation (4.13) says

$$
\frac{\partial}{\partial z_{1}} \boldsymbol{B} f(z)=\frac{1}{z_{1}} \int_{\left|w_{2}\right|=0}^{\left|w_{2}\right|=1}\left\{\int_{\mathcal{D}} \mathcal{T}_{w_{1}}(B(z, w)) f(w) d V\left(w_{1}\right)\right\} d V\left(w_{2}\right),
$$

where the inner integral is taken over $\mathcal{D}=\left\{w_{1}:\left|w_{1}\right|<\left|w_{2}\right|^{n}\right\}$ for each fixed $w_{2}$. Estimating this term requires more care than was necessary for the $z_{2}$ derivative. As in the proof of Lemma 2.12, define a kernel by subtracting from $B(z, w)$ the term $B\left(\left(0, z_{2}\right),\left(0, w_{2}\right)\right)$. Equation (4.11) shows

$$
\begin{aligned}
K(z, w) & :=B(z, w)-B\left(\left(0, z_{2}\right),\left(0, w_{2}\right)\right) \\
& =\frac{1}{\pi^{2}}\left[\frac{z_{2}^{n} \bar{w}_{2}^{n}}{\left(1-z_{2} \bar{w}_{2}\right)^{2}\left(z_{2}^{n} \bar{w}_{2}^{n}-z_{1} \bar{w}_{1}\right)^{2}}-\frac{1}{z_{2}^{n} \bar{w}_{2}^{n}\left(1-z_{2} \bar{w}_{2}\right)^{2}}\right] \\
& =\frac{1}{\pi^{2}} \frac{2 z_{1} \bar{w}_{1} z_{2}^{n} \bar{w}_{2}^{n}-z_{1}^{2} \bar{w}_{1}^{2}}{z_{2}^{n} \bar{w}_{2}^{n}\left(1-z_{2} \bar{w}_{2}\right)^{2}\left(z_{2}^{n} \bar{w}_{2}^{n}-z_{1} \bar{w}_{1}\right)^{2}} .
\end{aligned}
$$

Since $B\left(\left(0, z_{2}\right),\left(0, w_{2}\right)\right)$ is independent of $w_{1}$ and $\bar{w}_{1}, K(z, w)$ may be substituted for $B(z, w)$ in equation (4.16). Since $\mathcal{D}$ is a disc centered at the origin, Proposition 2.11 applies:

$$
\begin{aligned}
(4.16) & =-\frac{1}{z_{1}} \int_{\left|w_{2}\right|=0}^{\left|w_{2}\right|=1}\left\{\int_{\mathcal{D}} K(z, w) \mathcal{T}_{w_{1}} f(w) d V\left(w_{1}\right)\right\} d V\left(w_{2}\right) \\
& =-\frac{1}{z_{1}} \int_{\mathbb{H}_{1 / n}} K(z, w) \mathcal{T}_{w_{1}} f(w) d V(w) \\
& =\int_{\mathbb{H}_{1 / n}} \frac{w_{1}}{z_{1}} K(z, w) \frac{\partial f}{\partial w_{1}}(w) d V(w)-\int_{\mathbb{H}_{1 / n}} \frac{\bar{w}_{1}}{z_{1}} K(z, w) \frac{\partial f}{\partial \bar{w}_{1}}(w) d V(w),
\end{aligned}
$$

derivatives interpreted distributionally, as before. By hypothesis, the functions $\frac{\partial f}{\partial w_{1}}, \frac{\partial f}{\partial \bar{w}_{1}} \in$ $L^{p}\left(\mathbb{H}_{1 / n}\right)$. 
From (4.17), the kernels in (4.18) satisfy

$$
\begin{aligned}
\left|\frac{w_{1}}{z_{1}} K(z, w)\right|=\left|\frac{\bar{w}_{1}}{z_{1}} K(z, w)\right| & \approx\left|\frac{w_{1}}{z_{1}}\right| \cdot \frac{\left|2 z_{1} \bar{w}_{1} z_{2}^{n} \bar{w}_{2}^{n}-z_{1}^{2} \bar{w}_{1}^{2}\right|}{\left|z_{2}\right|^{n}\left|\bar{w}_{2}\right|^{n}\left|1-z_{2} \bar{w}_{2}\right|^{2}\left|z_{2}^{n} \bar{w}_{2}^{n}-z_{1} \bar{w}_{1}\right|^{2}} \\
& \lesssim\left|\frac{w_{1}}{z_{1}}\right| \cdot \frac{\left|z_{1}\right|\left|w_{1}\right|\left|z_{2}\right|^{n}\left|w_{2}\right|^{n}}{\left|z_{2}\right|^{n}\left|w_{2}\right|^{n}\left|1-z_{2} \bar{w}_{2}\right|^{2}\left|z_{2}^{n} \bar{w}_{2}^{n}-z_{1} \bar{w}_{1}\right|^{2}} \\
& \leq \frac{\left|w_{2}\right|^{2 n}}{\left|1-z_{2} \bar{w}_{2}\right|^{2}\left|z_{2}^{n} \bar{w}_{2}^{n}-z_{1} \bar{w}_{1}\right|^{2}} .
\end{aligned}
$$

The last two inequalities hold because $z, w \in \mathbb{H}_{1 / n}$. Lemma 4.1, with $c=0, d=2 n$, and $m=1$, shows

$$
\left\|\frac{\partial}{\partial z_{1}} \circ \boldsymbol{B} f\right\|_{L^{p}\left(\mathbb{H}_{1 / n}\right)} \lesssim\|f\|_{L_{1}^{p}\left(\mathbb{H}_{1 / n}\right)}
$$

for $p \in\left(1, \frac{2 n+2}{2 n}\right)$, establishing part (1) of the theorem.

Corollary 4.19. The Bergman projection $\boldsymbol{B}$ is a bounded operator from $L_{1}^{p}\left(\mathbb{H}_{1}\right) \rightarrow L_{1}^{p}\left(\mathbb{H}_{1}\right)$, for all $\frac{4}{3}<p<2$.

Proof. Set $n=1$ in Theorem 4.12 and intersect the two intervals of $L^{p}$ boundedness. It follows that $D \circ \boldsymbol{B}$ is $L^{p}$ bounded for $1<p<2$ for any first derivative $D$. Since $\boldsymbol{B}$ itself is $L^{p}$ bounded for $\frac{4}{3}<p<4$ (Theorem [3.1), the result follows.

\section{A substitute operator on the Hartogs triangle}

In light of Theorem [1.3, it is natural to seek operators related to $\boldsymbol{B}$ which have better Sobolev mapping behavior than $\boldsymbol{B}$ itself. Pursuing an idea in [10, a sub-Bergman operator is constructed on $\mathbb{H}_{1}$ with such improved behavior. $\mathbb{H}_{1}$ is taken only for simplicity; the general pattern below extends to other domains.

Consider the set of bounded monomials on $\mathbb{H}_{1}$ :

$$
\mathcal{S}\left(\mathbb{H}_{1}, L^{\infty}\right)=\left\{\alpha=\left(\alpha_{1}, \alpha_{2}\right): \alpha_{1} \geq 0, \quad \alpha_{1}+\alpha_{2} \geq 0\right\} .
$$

Lemma 3.2 shows that $\mathcal{S}\left(\mathbb{H}_{1}, L^{\infty}\right)=\mathcal{S}\left(\mathbb{H}_{1}, L^{p}\right)$ for $p \geq 4$ and $\mathcal{S}\left(\mathbb{H}_{1}, L^{\infty}\right) \subsetneq \mathcal{S}\left(\mathbb{H}_{1}, L^{2}\right)$. Following [10, define the $L^{\infty}$ sub-Bergman kernel

$$
\widetilde{B^{\infty}}(z, w):=\sum_{\alpha \in \mathcal{S}\left(\mathbb{H}_{1}, L^{\infty}\right)} \frac{z^{\alpha} \bar{w}^{\alpha}}{\left\|z^{\alpha}\right\|_{L^{2}\left(\mathbb{H}_{1}\right)}^{2}} .
$$

Notice the series in (5.1) is only part of the usual series that defines the Bergman kernel. The $L^{\infty}$ sub-Bergman projection is

$$
\widetilde{\boldsymbol{B}^{\infty}} f(z):=\int_{\mathbb{H}_{1}} \widetilde{B^{\infty}}(z, w) f(w) d V(w)
$$

whenever the integral converges; $f$ is taken from certain $L_{k}^{p}\left(\mathbb{H}_{1}\right)$ classes below.

A rational expression for (5.1) follows from [10, Proposition 4.33]:

$$
\widetilde{B^{\infty}}(z, w)=\frac{1}{\pi^{2}} \frac{2 z_{2}^{2} \bar{w}_{2}^{2}-z_{2}^{3} \bar{w}_{2}^{3}}{\left(z_{2} \bar{w}_{2}-z_{1} \bar{w}_{1}\right)^{2}\left(1-z_{2} \bar{w}_{2}\right)^{2}} .
$$

This immediately yields the bound

$$
\left|\widetilde{B^{\infty}}(z, w)\right| \lesssim \frac{\left|z_{2}\right|^{2}\left|w_{2}\right|^{2}}{\left|z_{2} \bar{w}_{2}-z_{1} \bar{w}_{1}\right|^{2}\left|1-z_{2} \bar{w}_{2}\right|^{2}} .
$$

Lemma 4.1 with $m=n=1$ and $c=d=2$ shows for each fixed $1<p<\infty$,

$$
\left\|\widetilde{\boldsymbol{B}^{\infty}} f\right\|_{L^{p}\left(\mathbb{H}_{1}\right)} \lesssim\|f\|_{L^{p}\left(\mathbb{H}_{1}\right)}, \quad f \in L^{p}\left(\mathbb{H}_{1}\right) .
$$


Derivatives are now considered. Mapping properties of $\frac{\partial}{\partial z_{2}} \circ \widetilde{\boldsymbol{B}^{\infty}}$ may be obtained by following the proof of Theorem 4.12 with $\widetilde{B^{\infty}}(z, w)$ replacing $B(z, w)$. The steps leading up to (4.15) show, for $f \in L_{1}^{p}\left(\mathbb{H}_{1}\right)$,

$$
\frac{\partial}{\partial z_{2}} \widetilde{\boldsymbol{B}^{\infty}} f(z)=\int_{\mathbb{H}_{1}} \frac{w_{2}}{z_{2}} \widetilde{B^{\infty}}(z, w) \frac{\partial f}{\partial w_{2}}(w) d V(w)-\int_{\mathbb{H}_{1}} \frac{\bar{w}_{2}}{z_{2}} \widetilde{B^{\infty}}(z, w) \frac{\partial f}{\partial \bar{w}_{2}}(w) d V(w) .
$$

Thus the operator $\frac{\partial}{\partial z_{2}} \circ \widetilde{\boldsymbol{B}^{\infty}}$ is controlled by the kernels

$$
\left|\frac{w_{2}}{z_{2}} \widetilde{B^{\infty}}(z, w)\right|=\left|\frac{\bar{w}_{2}}{z_{2}} \widetilde{B^{\infty}}(z, w)\right| \lesssim \frac{\left|z_{2}\right|\left|w_{2}\right|^{3}}{\left|z_{2} \bar{w}_{2}-z_{1} \bar{w}_{1}\right|^{2}\left|1-z_{2} \bar{w}_{2}\right|^{2}} .
$$

Lemma 4.1 (and Remark 4.5) with $m=n=1, c=1, d=3$, shows for each fixed $1<p<4$,

$$
\left\|\frac{\partial}{\partial z_{2}} \circ \widetilde{\boldsymbol{B}^{\infty}} f\right\|_{L^{p}\left(\mathbb{H}_{1}\right)} \lesssim\left\|\frac{\partial f}{\partial w_{2}}\right\|_{L^{p}}+\left\|\frac{\partial f}{\partial \bar{w}_{2}}\right\|_{L^{p}} \leq\|f\|_{L_{1}^{p}\left(\mathbb{H}_{1}\right)} .
$$

Mapping properties of $\frac{\partial}{\partial z_{1}} \circ \widetilde{\boldsymbol{B}^{\infty}}$ may be obtained by considering

$$
\begin{aligned}
\widetilde{K^{\infty}}(z, w) & :=\widetilde{B^{\infty}}(z, w)-\widetilde{B^{\infty}}\left(\left(0, z_{2}\right),\left(0, w_{2}\right)\right) \\
& =\frac{1}{\pi^{2}}\left[\frac{2 z_{2}^{2} \bar{w}_{2}^{2}-z_{2}^{3} \bar{w}_{2}^{3}}{\left(1-z_{2} \bar{w}_{2}\right)^{2}\left(z_{2} \bar{w}_{2}-z_{1} \bar{w}_{1}\right)^{2}}-\frac{2 z_{2}^{2} \bar{w}_{2}^{2}-z_{2}^{3} \bar{w}_{2}^{3}}{z_{2}^{2} \bar{w}_{2}^{2}\left(1-z_{2} \bar{w}_{2}\right)^{2}}\right] \\
& =\frac{1}{\pi^{2}} \frac{z_{1} \bar{w}_{1}\left(4 z_{2} \bar{w}_{2}-2 z_{2}^{2} \bar{w}_{2}^{2}-2 z_{1} \bar{w}_{1}+z_{1} \bar{w}_{1} z_{2} \bar{w}_{2}\right)}{\left(1-z_{2} \bar{w}_{2}\right)^{2}\left(z_{2} \bar{w}_{2}-z_{1} \bar{w}_{1}\right)^{2}} .
\end{aligned}
$$

Simple estimation shows $\widetilde{K^{\infty}}(z, w)$ satisfies a stronger estimate than (5.3):

$$
\left|\widetilde{K^{\infty}}(z, w)\right| \lesssim \frac{\left|z_{1}\right|\left|w_{1}\right|\left|z_{2}\right|\left|w_{2}\right|}{\left|1-z_{2} \bar{w}_{2}\right|^{2}\left|z_{2} \bar{w}_{2}-z_{1} \bar{w}_{1}\right|^{2}}
$$

Repeating the steps from (4.16) through (4.18) - with $\widetilde{K^{\infty}}(z, w)$ replacing $K(z, w)$ - shows, for $f \in L_{1}^{p}\left(\mathbb{H}_{1}\right)$,

$$
\frac{\partial}{\partial z_{1}} \widetilde{\boldsymbol{B}^{\infty}} f(z)=\int_{\mathbb{H}_{1}} \frac{w_{1}}{z_{1}} \widetilde{K^{\infty}}(z, w) \frac{\partial f}{\partial w_{1}}(w) d V(w)-\int_{\mathbb{H}_{1}} \frac{\bar{w}_{1}}{z_{1}} \widetilde{K^{\infty}}(z, w) \frac{\partial f}{\partial \bar{w}_{1}}(w) d V(w) .
$$

Thus the operator $\frac{\partial}{\partial z_{1}} \circ \widetilde{\boldsymbol{B}^{\infty}}$ is controlled by the kernels

$$
\left|\frac{w_{1}}{z_{1}} \widetilde{K^{\infty}}(z, w)\right|=\left|\frac{\bar{w}_{1}}{z_{1}} \widetilde{K^{\infty}}(z, w)\right| \lesssim \frac{\left|z_{2}\right|\left|w_{2}\right|^{3}}{\left|1-z_{2} \bar{w}_{2}\right|^{2}\left|z_{2} \bar{w}_{2}-z_{1} \bar{w}_{1}\right|^{2}} .
$$

This bound is identical to the bound in (5.5). Consequently, for each fixed $1<p<4$,

$$
\left\|\frac{\partial}{\partial z_{1}} \circ \widetilde{\boldsymbol{B}^{\infty}} f\right\|_{L^{p}\left(\mathbb{H}_{1}\right)} \lesssim\left\|\frac{\partial f}{\partial w_{1}}\right\|_{L^{p}}+\left\|\frac{\partial f}{\partial \bar{w}_{1}}\right\|_{L^{p}} \leq\|f\|_{L_{1}^{p}\left(\mathbb{H}_{1}\right)} .
$$

Combining (5.4), (5.6), and (5.7) proves the following

Corollary 5.8. $\widetilde{\boldsymbol{B}^{\infty}}$ maps $L_{1}^{p}\left(\mathbb{H}_{1}\right) \rightarrow L_{1}^{p}\left(\mathbb{H}_{1}\right)$ boundedly for all $1<p<4$.

It is not difficult to verify that $\widetilde{\boldsymbol{B}^{\infty}}$ fails to map $L_{1}^{p}\left(\mathbb{H}_{1}\right) \rightarrow L_{1}^{p}\left(\mathbb{H}_{1}\right)$ for $p \geq 4$ : take the monomial $f(z)=z_{1} \bar{z}_{2}$ and follow the arguments given in Section 3. The interested reader is invited to extend Corollary 5.8 to higher order derivatives. The statements are

Corollary 5.9. $\widetilde{\boldsymbol{B}^{\infty}}$ maps $L_{2}^{p}\left(\mathbb{H}_{1}\right) \rightarrow L_{2}^{p}\left(\mathbb{H}_{1}\right)$ boundedly for all $1<p<2$.

Corollary 5.10. $\widetilde{\boldsymbol{B}^{\infty}}$ maps $L_{3}^{p}\left(\mathbb{H}_{1}\right) \rightarrow L_{3}^{p}\left(\mathbb{H}_{1}\right)$ boundedly for all $1<p<\frac{4}{3}$. 
Remark 5.11. Formulas (5.1) and (5.2) can be modified to define the $L^{\infty}$ sub-Bergman kernel and projection on a general Reinhardt domain $\mathcal{R}$. More generally, for fixed $p \in[2, \infty), L^{p}$ sub-Bergman kernels and projections $\left(\widetilde{B^{p}}(z, w)\right.$ and $\left.\widetilde{\boldsymbol{B}^{p}}\right)$ may be defined on $\mathcal{R}$ by formulas analogous to (5.1), where the sum is taken over indices $\alpha \in \mathcal{S}\left(\mathcal{R}, L^{p}\right)$ - see [10, Section 3.6].

In [10, Section 4.2.2], the $\widetilde{\boldsymbol{B}^{p}}$ are constructed for each $\mathbb{H}_{m / n}$ and shown to stabilize into $m+n$ representatives. These operators are more regular on $L_{0}^{p}$ than $\boldsymbol{B}$ is - see [10, Theorem 4.3]. This improved regularity has consequences for holomorphic duality and approximation - see [10, Section 4.4].

\section{ACKNOWLEDGMENTS}

The authors thank the Erwin Schrödinger Institute, Vienna for providing us a fruitful environment for collaboration during a December 2018 workshop. The first author also thanks Texas A\&M at Qatar for hosting the stimulating workshop Analysis and Geometry in Several Complex Variables III in January 2019.

The authors are also grateful to the two anonymous referees, whose comments improved the mathematical and expository content of the paper.

\section{REFERENCES}

[1] BARrett, D., AND ŞAhutoĞLu, S. Irregularity of the Bergman projection on worm domains in $\mathbb{C}^{n}$. Michigan Math. J. 61, 1 (2012), 187-198.

[2] Barrett, D. E. Irregularity of the Bergman projection on a smooth bounded domain in $\mathbf{C}^{2}$. Ann. of Math. (2) 119, 2 (1984), 431-436.

[3] Barrett, D. E. Regularity of the Bergman projection and local geometry of domains. Duke Math. J. 53, 2 (1986), 333-343.

[4] Barrett, D. E. Behavior of the Bergman projection on the Diederich-Fornæss worm. Acta Math. 168, 1-2 (1992), 1-10.

[5] Bell, S., ANd LigockA, E. A simplification and extension of Fefferman's theorem on biholomorphic mappings. Invent. Math. 57, 283-289 (1980).

[6] Bell, S. R. Biholomorphic mappings and the $\bar{\partial}$-problem. Ann. of Math. (2) 114, 1 (1981), 103-113.

[7] Boas, H. P. The Szegő projection: Sobolev estimates in regular domains. Trans. Amer. Math. Soc. 300, 1 (1987), 109-132.

[8] Boas, H. P., And Straube, E. J. Sobolev estimates for the $\bar{\partial}$-Neumann operator on domains in $\mathbf{C}^{n}$ admitting a defining function that is plurisubharmonic on the boundary. Math. Z. 206, 1 (1991), 81-88.

[9] Boas, H. P., And Straube, E. J. Global regularity of the $\bar{\partial}$-Neumann problem: a survey of the $L^{2}$-Sobolev theory. In Several complex variables (Berkeley, CA, 1995-1996), vol. 37 of Math. Sci. Res. Inst. Publ. Cambridge Univ. Press, Cambridge, 1999, pp. 79-111.

[10] Chakrabarti, D., Edholm, L. D., and McNeal, J. D. Duality and approximation of Bergman spaces. Adv. Math. 341 (2019), 616-656.

[11] Chakrabarti, D., And Zeytuncu, Y. $L^{p}$ mapping properties of the Bergman projection on the Hartogs triangle. Proc. Amer. Math. Soc. 144, 4 (2016), 1643-1653.

[12] Charpentier, P., And Dupain, Y. Estimates for the Bergman and Szegö projections on pseudoconvex domains of finite type with locally diagonalizable Levi form. Publ. Math. 50, 2 (2006), 413-446.

[13] Chen, L. The $L^{p}$ boundedness of the Bergman projection for a class of bounded Hartogs domains. $J$. Math. Anal. Appl. 448, 1 (2017), 598-610.

[14] Chen, L. Weighted Sobolev regularity of the Bergman projection on the Hartogs triangle. Pacific J. Math. 288 (2017), 307-318.

[15] Detraz, J. Classes de Bergman de fonctions harmoniques. Bull. Soc. Math. France 109 (1981), 259268.

[16] Diederich, K., And Fornæss, J. E. Pseudoconvex domains: an example with nontrivial Nebenhülle. Math. Ann. 225, 3 (1977), 275-292.

[17] Duren, P., And Schuster, A. Bergman spaces, vol. Mathematical surveys and mongraphs. American Mathematical Society, 2004.

[18] Edholm, L. D. Bergman theory of certain generalized Hartogs triangles. Pacific J. Math. 284, 2 (2016), $327-342$. 
[19] Edholm, L. D., And McNeal, J. D. The Bergman projection on fat Hartogs triangles: $L^{p}$ boundedness. Proc. Amer. Math. Soc. 144, 5 (2016), 2185-2196.

[20] Edholm, L. D., AND McNeal, J. D. Bergman subspaces and subkernels: degenerate $L^{p}$ mapping and zeroes. J. Geom. Anal. 27, 4 (2017), 2658-2683.

[21] Forelli, F., And Rudin, W. Projections on spaces of holomorphic functions in balls. Ind. Univ. Math. J. 24 (1974), 593-602.

[22] Herbig, A.-K., And McNeal, J. A smoothing property of the Bergman projection. Math. Ann. 354, 2 (2012), 427-449.

[23] Koenig, K. D. On maximal Sobolev and Hölder estimates for the tangential Cauchy-Riemann operator and boundary Laplacian. Amer. J. Math. 124, 1 (2002), 129-197.

[24] Koнn, J. J. Quantitative estimates for global regularity. In Analysis and geometry in several complex variables (Katata, 1997), Trends Math. Birkhäuser Boston, Boston, MA, 1999, pp. 97-128.

[25] McNeal, J. D. Boundary behavior of the Bergman kernel function in $\mathbf{C}^{2}$. Duke Math. J. 58, no. 2 (1989), 499-512.

[26] McNeal, J. D. Local geometry of decoupled pseudoconvex domains. In Complex analysis (Wuppertal, 1991), Aspects Math., E17. Vieweg, Braunschweig, 1991, pp. 223-230.

[27] McNeal, J. D. Estimates on the Bergman kernels of convex domains. Adv. Math. 109, 1 (1994), $108-139$.

[28] McNeal, J. D., And Stein, E. M. Mapping properties of the Bergman projection on convex domains of finite type. Duke Math. J. 73, 1 (1994), 177-199.

[29] McNeal, J. D., And Stein, E. M. The Szegö projection on convex domains. Math. Z. 224, 4 (1997), $519-553$.

[30] Nagel, A., Rosay, J.-P., Stein, E. M., And Wainger, S. Estimates for the Bergman and Szego kernels in $\mathbf{C}^{2}$. Ann. of Math. (2) 129, 1 (1989), 113-149.

[31] Phong, D. H., And Stein, E. M. Estimates for the Bergman and Szegö projections on strongly pseudo-convex domains. Duke Math. J. 44, 3 (1977), 695-704.

[32] Rudin, W. Function theory in the unit ball in $\mathbb{C}^{n}$, vol. 241 of Grundlehren der Mathematischen Wissenschaften. Springer-Verlag, New York, 1980.

[33] Zaharjuta, V., And Judovic, V. The general form of a linear functional on $H_{p}^{\prime}$. Uspekhi Mat. Nauk. 19, 2 (1964), 139-142.

[34] Zeytuncu, Y. $L^{p}$ regularity of weighted Bergman projections. Trans. Amer. Math. Soc. 365, no. 6 (2013), 2959-2976.

[35] ZHu, K. Spaces of holomorphic functions in the unit ball, vol. 226 of Graduate Texts in Mathematics. Springer-Verlag, New York, 2005.

Department of Mathematics,

University of Michigan, Ann Arbor, Michigan, USA

E-mail address: edholm@umich.edu

Department of Mathematics,

The Ohio State University, Columbus, Ohio, USA

E-mail address: mcneal@math.ohio-state.edu 